\title{
Las Actividades que Conforman el Rol del Director de Facultad
}

\author{
John Farrand Rogers \\ Facultad de Planeación Urbana-UAEM
}

Resumen: Se presentan los resultados de un análisis de los datos arrojados por un estudio sobre el rol del director de facultad. Se encuentra que su ubicación según 100 categorías de actividad resulta inadecuada para explicar claramente todo el fenómeno; hace falta contemplar también otras dimensiones.

Abstract: This essay is a synthetic exposition about the findings of a large research about the role playing theory, applying to understand attitudes and role playing the principal manager of a faculty at University. From evaluating 100 variables, that where organized into twenty categories the author analyzes the social role of the faculty director.

$\square_{\text {al director de facultad. }}^{\text {n este análisis se presenta alguna información que emergió de un }}$

Esencialmente, este proyecto pretende profundizar algunos aspectos de esta teoría, sobre todo los que puedan ser examinados a través de una investigación de campo. Para el caso específico de estudio, se aplica al puesto de director de facultad, debido a que no se encuentran muchos estudios realizados sobre éste, aunque sea tan importante en la vida universitaria (García y Mejía, 1990; Farrand, 1993); y en parte porque aun una observación superficial deja ver que éste tiene algunas diferencias muy importantes frente a un puesto correspondiente dentro de la universidad de algunos otros países.

Evidentemente, en este momento no se pretende profundizar en todos los detalles, porque el lugar para hacerlo es el reporte final. Lo que nos interesa ahora es el análisis de los resultados del trabajo de

1 Este proyecto se lleva a cabo con el respaldo de la Coordinación General de Investigación y Estudios Avanzados de la Universidad Autónoma del Estado de México. 
campo, para ver si se puede distinguir algún patrón emergente, que oriente y profundice nuestra comprensión del objeto de estudio. En este trabajo, entonces, se toma como patrón para organizar el análisis a las 20 categorías de posibles actividades que dirigieron la construcción del instrumento.

No obstante, para mayor comprensión empezamos abordando, aunque de manera muy breve, algunos detalles importantes: un acercamiento a la teoría de rol y algunas cuestiones metodológicas, para enfocar finalmente con más detalle algunos de los hallazgos sustantivos.

\section{La teoría de rol}

La teoría de rol tuvo sus orígenes en los años cincuenta, de manera que actualmente se encuentra bastante consolidada a nivel mundial, con sus propios conceptos y técnicas establecidas (Gross et al, 1958; Banton, 1965; Biddle y Thomas, 1966; Popitz, 1972). Esta teoría deriva de la idea de roles en el mundo del teatro, donde existe una distinción entre el rol y el actor que lo representa (Hargreaves, 1972). Cuando observamos algún rol conocido dentro de una obra de teatro, podemos distinguir entre lo medular (lo que los actores necesariamente tienen que hacer $\mathrm{u}$ omitir) y lo discrecional (lo que pueden hacer $\mathrm{u}$ omitir de acuerdo con su propia interpretación). De esta manera podemos calificar como excelente o inadecuada cualquier representación.

Lo mismo pasa en el mundo social, donde los seres humanos (como "actores") representamos en diferentes momentos los diversos papeles (o roles) que nos corresponden. Todos podemos reconocer el rol social del policía, del profesor, del padre de familia, del sacerdote. Estos roles tienen elementos que necesariamente están presentes para que los reconozcamos. Al mismo tiempo, todos nos extrañaríamos si el sacerdote se comportara como futbolista, o el profesor como policía. Tales conductas no serían congruentes con su rol.

Estos roles son configurados por las expectativas que tenemos, las cuales modifican constantemente la interpretación de cualquier actor, quien las toma en cuenta, sobre todo las de quienes tienen mayor importancia para su actualización, específicamente sus superiores jerárquicos. Por lo tanto, la definición de cualquier rol llega a ser resultado de un acto social. Aunque finalmente es el propio actor quien 
toma la decisión acerca de cómo debe ser su representación, lo hace bajo la influencia de todos los involucrados con su posición social y ésta puede modificar su conducta cuando los demás lo vemos como incongruente. Si no la toma en cuenta, puede encontrar un rechazo ante su actuación, la cual imposibilita el éxito en sus actividades (Hargreaves, 1972).

Si tomamos el ejemplo de un director de facultad, podemos pensar en los profesores, los alumnos, los trabajadores y también en los funcionarios del edificio central como influyentes en la interpretación del rol (Ortiz y Farrand, 1994). Por supuesto, la definición oficial que encuentra su expresión en los reglamentos y la precisión jurídica de los atributos, derechos y obligaciones de un director, es una influencia muy importante. No podemos dejar de tomar en cuenta la orientación oficial (véase Verona, 1996, por ejemplo). Pero en la práctica, un director puede llevar a cabo con éxito otras actividades (más que las definidas en el reglamento, o menos), siempre que esté actuando dentro de su rol social, recibiendo una aceptación y legitimación por parte de los demás.

Hay que aclarar que las acciones del director que se mencionan a continuación, no son las que corresponden con sus responsabilidades, porque éstas están descritas detalladamente dentro del Estatuto de la Universidad; solamente son las acciones que corresponden con las expectativas de los tres grupos de informantes. Asimismo, hay que aclarar que las acciones que se describen en el instrumento aplicado son las que cuentan con cierto grado de ambigüedad, en el sentido de que pueden ser aceptables o no. Es este tipo de acción que define los límites del rol.

\section{Cuestiones metodológicas}

Para conformar el cuestionario, cuyos resultados se presentan en este espacio, se acudió primero a un análisis basado en un repaso de la literatura sobre la actividad del director de escuela secundaria, en primer momento en Inglaterra (donde es un tema bastante estudiado), y modificado posteriormente en el campo para el caso de México (Farrand, 1987). Este análisis proporcionó un panorama bastante completo de las diferentes actividades que integran la dirección de una escuela en términos generales, y sirvió como guión para entrevistar a diez informantes (cuatro alumnos y seis profesores o funcionarios de la universidad). Los aspectos que no encontraron eco en las entrevistas 
fueron descartados, mientras otros detalles que surgieron en la entrevista fueron incorporados. De esta manera, los temas a abordar fueron afinados cada vez más para enfocarse en el objeto de estudio.

En esa etapa, nuestros informantes fueron seleccionados, en parte, por sus conocimientos del funcionamiento de la universidad mexicana y el desempeño de sus directores de facultad; así como por su reconocida actitud académicamente crítica frente a cuestiones políticas y sociales; de manera que podemos identificar a este grupo como informantes clave. ${ }^{2}$

Con base en la información arrojada por las entrevistas, se diseñó como el paso siguiente unos 600 reactivos, de acuerdo con un modelo de Likert, donde los encuestados tienen que expresar qué tanto están de acuerdo con cada reactivo, en una escala de cinco puntos, desde una aceptación total hasta un rechazo total. Este número fue reducido por la eliminación de los que parecían duplicar temas; los que conducían a respuestas muy evidentes acerca de actividades obligatorias de acuerdo con la reglamentación universitaria vigente (Verona, 1996) o de actividades prohibidas moral o legalmente (Farrand, 1996); y por la integración de dos ítems en uno solo para aumentar el grado de dificultad del reactivo. El piloteo del cuestionario tanto, con alumnos como con profesores, sirvió para eliminar o mejorar la redacción de algunos reactivos que provocaron dudas.

El instrumento final de 100 reactivos se aplicó a una muestra de 216 alumnos en el tercer año de sus estudios; y a 87 profesores de tiempo completo de cinco facultades de la universidad, las cuales cubrían una gama de disciplinas y profesiones (derecho, ciencias de la conducta, arquitectura, medicina y planeación urbana y regional). Además se les aplicó a todos los directores vigentes de la universidad, y a todos menos a dos de sus precursores que habían ocupado el puesto en los cinco años anteriores, dando un total de 43 directores encuestados.

Posteriormente, se les asignó puntos a sus respuestas (de acuerdo con el grado de aceptación), los cuales se sumaron para calcular la media para cada grupo de encuestados y las desviaciones estándar.

2 En esta parte del estudio participó Gumaro Verona Casas, como auxiliar de investigación. 
Como el número de informantes de cada grupo era diferente, se procedió a calcular las medias de las medias y de las desviaciones, de manera que las respuestas de los tres grupos de alumnos, profesores y directores tuvieran el mismo peso al determinar cifras globales para todos los encuestados. Estas son las cifras que se consideran dentro del análisis actual. $^{3}$

Para interpretar las medias aquí presentadas, habrá que señalar que un cinco implica una fuerte aceptación de lo propuesto por el reactivo, mientras que un uno significa un fuerte rechazo; un cuatro y un dos implican lo mismo, pero en menor grado. La dirección de la respuesta (positiva o negativa) no es en sí muy importante, porque depende de la redacción de cada reactivo. En contraste, cuando una media se presenta como un tres, implica que ese reactivo no tiene dirección, quedándose en la ambigüedad; ésta se puede atribuir a una de dos causas: o existe un consenso entre los informantes en el sentido de que la acción expresada por el reactivo no tiene importancia dentro de la definición del rol del director; o que las respuesta divergen mucho, por lo cual el punto medio disfraza una falta de consenso. Esta convergencia o divergencia entre los informantes es representada por las desviaciones estándar, donde una cifra menor de 1.0 representa bastante consenso, mientras que las cifras más elevadas representan su ausencia.

Para interpretar las medias aquí presentadas, habrá que señalar que un cinco implica una fuerte aceptación de lo propuesto por el reactivo, mientras que un uno significa un fuerte rechazo; un cuatro y un dos implican lo mismo, pero en menor grado. La dirección de la respuesta (positiva o negativa) no es en sí muy importante, porque depende de la redacción de cada reactivo. En contraste, cuando una media se presenta como un tres, implica que ese reactivo no tiene dirección, quedándose en la ambigüedad; ésta se puede atribuir a una de dos causas: o existe un consenso entre los informantes en el sentido de que la acción expresada por el reactivo no tiene importancia dentro de la definición del rol del director; o que las respuesta divergen mucho, por lo cual el punto medio disfraza una falta de consenso. Esta

3 Quisiéramos agradecer la contribución del Centro de Servicios de Cómputo de la UAEM, y sobre todo a Luis Enrique López Díaz, por su apoyo en esta parte del proyecto. 
convergencia o divergencia entre los informantes es representada por las desviaciones estándar, donde una cifra menor de 1.0 representa bastante consenso, mientras que las cifras más elevadas representan su ausencia.

A continuación se presentan los datos de acuerdo con las 20 categorías de actividades que se identificaron en la literatura sobre la dirección de escuelas secundarias. Dos maneras alternativas para presentar esta información son a través de un ordenamiento de las respuestas de acuerdo con sus medias: así se puede ver cuáles acciones tienen carácter obligatorio y cuáles son solamente recomendables para el rol del director. La otra alternativa es su análisis desde el punto de vista de las desviaciones estándar, por lo cual podemos ver dónde existe mayor consenso. Se pretende que estas otras dos maneras de analizar los datos se presenten en algún momento y en otro espacio.

\section{Reactivos organizados por categorías de acciones}

Así es que en este espacio iniciamos el análisis repartiendo los reactivos según las categorías que empleamos en las entrevistas, tales como administración, comunicación interna, trabajo fuera de la facultad, todas de las cuales se presentan abajo en el espacio correspondiente (identificadas con las letras A, B, C, etc.). Estas categorías son construidas para la conveniencia de análisis, y puede ser que otros analistas prefieran ubicar ciertos ítems en una categoría diferente a la que hemos usado.

Las tablas que siguen se refieren a las 20 categorías de actividades. Se notará en seguida que no hay un equilibrio entre las categorías, algunas tienen más reactivos que otras. Esto se explica por la manera en que conformamos el instrumento. Esencialmente, las categorías eran derivadas de estudios sobre la dirección de escuelas secundarias en general a nivel internacional, de manera que algunas no van a tener la misma importancia cuando se habla acerca de la dirección de una facultad en México. El filtro que funcionó para dar más o menos peso a cada categoría, lo fueron las entrevistas con los informantes clave, de esta forma sería justificable definir la importancia de las categorías de acuerdo con el número de reactivos que contengan.

Con base en esto, se verá que las categorías con mayor número de reactivos son $\mathrm{M}$ (comunicación interna) y $\mathrm{T}$ (relaciones con instituciones externas), cada una con nueve; siguen B (currículum 
académico), E (recursos) G (personal docente) y P (responsabilidad externa/institucional); éstas cuentan con ocho reactivos cada una.

En contraste, dos categorías cuentan solamente con un reactivo, de manera que su inclusión en la lista parece ser un poco simbólica: éstas son $\mathrm{C}$ (currículum para la orientación de alumnos) y $\mathrm{S}$ (trabajo fuera de la facultad).

\begin{tabular}{|c|c|c|}
\hline (1) & 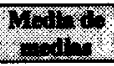 & (1), \\
\hline $\begin{array}{l}\text { 1. Asumir como su propia responsabilidad los aspectos políticos de la Universidad } \\
\text { más que los aspectos académicos y cientlficos. }\end{array}$ & 3.02 & 1.46 \\
\hline 2. Definir metas precisas y claras con los tiempos para alcanzarios. & 4.82 & 0.49 \\
\hline 3. Dedicarse sólo a administrar los recursos. & 2.36 & 1.33 \\
\hline $\begin{array}{l}\text { 4. Alcanzar las metas institucionales, aun acudiendo a prácticas impositivas o } \\
\text { explotando relaciones de amistad. }\end{array}$ & 2.33 & 1.41 \\
\hline $\begin{array}{l}\text { 5. Aprovecharse de los problemas casuales para ganar avances en el proyecto } \\
\text { institucional. }\end{array}$ & 2.32 & 1.46 \\
\hline
\end{tabular}

En este primer bloque de actividades se ve que la definición de metas (2) cuenta con un apoyo abrumante por parte de los encuestados, quienes además demuestran un elevado grado de consenso, como la desviación estándar es solamente de 0.49. Esta idea se refuerza con el rechazo significativo de la propuesta (3) de que el director debiera limitarse a la administración de recursos; lo cual implica el deseo que demuestre un liderazgo académico, el cual implica a su vez la búsqueda de metas.

Es un poco sorprendente, por lo tanto, que haya un rechazo antes las proposiciones 4 y 5 , que dan prioridad a la consecución de metas por encima de cualquier otra consideración. El rechazo del uso de cualquier mecanismo para alcanzar las metas (4) sugiere que cuestiones éticas también son importantes; sin embargo, el rechazo similar del aprovechamiento de problemas inmediatos para buscar soluciones que también apoyan el avance institucional (5) sugiere que tal vez los encuestados no vean la dirección como actividad compleja, sino que prefieran soluciones sencillas aplicadas claramente a cada problema.

La disparidad de respuestas ante el primer reactivo que pretende discriminar entre las actividades políticas (que pudieran incluir la precisión de objetivos a nivel universidad) y las académicas y administrativas (que se realizan más bien a nivel facultad), aparte de la amplia gama de respuestas indicadas por la elevada desviación 
estándar, demuestra una falta de claridad para el rol del director sobre esta dicotomía.

Lo que derivamos de estas respuestas es el deseo de que el director defina bien las metas institucionales, pero al mismo tiempo cierta renuencia que sea él solo quien defina los mecanismos.

\section{B. Curriculum Académico}

\begin{tabular}{|c|c|c|}
\hline (1) & 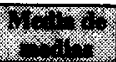 & Why \\
\hline 6. Poder enseffar el contenido de todas las materias. & 2.89 & 1.37 \\
\hline 7. Dejar ver que el contenido de la curricula tiene mucha importancia para él. & 3.96 & 1.18 \\
\hline 8. Conocer las nuevas tendencias en otros países para adecuar planes y programas. & 4.43 & 0.83 \\
\hline 9. Adecuar la curricula según el perfil de estudiante que requiere el mercado. & 4.41 & 0.91 \\
\hline $\begin{array}{l}\text { 10. Elaborar planes y programas respetando los tiempos administrativos, aun } \\
\text { sacrificando una amplia reflexión colectiva. }\end{array}$ & 2.81 & 1.28 \\
\hline 11. Revisar planes y programas cada semestre. & 4.26 & 1.10 \\
\hline $\begin{array}{l}\text { 12. Satisfacer las demandas de los alumnos, aun disminuyendo la exigencia } \\
\text { académica. }\end{array}$ & 2.09 & 1.10 \\
\hline 20. Tener grado avanzado de estudios. & 4.59 & 0.70 \\
\hline
\end{tabular}

Este grupo contiene un número bastante elevado de reactivos. Dos de ellos, que se refieren a la enseñanza de todas las materias (6) y el respeto a pautas administrativas para elaborar los planes (10), se ubican en el grado donde no importa si el director las haga o no; pero este resultado disfraza la elevada gama de respuestas. No existe ningún consenso sobre estos aspectos, en el primer caso a lo mejor porque los encuestados piensan de diferentes maneras, y no porque vean esta cuestión como algo sin importancia; y en el segundo caso, casi seguramente porque el reactivo se polariza entre el respecto a los tiempos y la conveniencia de consultar ampliamente. Ambas actividades son importantes (y sin duda, si se hubieran presentado por separado, cada una hubiera recibido apoyo), pero en este caso no se detecta una priorización para ninguna de las dos.

Casi todos los otros reactivos caen dentro de lo deseable más que lo obligatorio (aunque en un caso, en sentido negativo). Aquí se ve una importancia que se da al liderazgo del director en lo académico, sobre todo actualizando los contenidos de los planes de estudio (11) de acuerdo con las necesidades del mercado laboral (9) y las tendencias en otros países (8). Aquí se nota una dimensión importante del rol en la vinculación de la institución con espacios de afuera. El reactivo que hace alusión al grado de estudios del director (20) tiene carácter 
obligatorio y refuerza la importancia de la dimensión académica dentro del rol.

Los dos reactivos restantes hablan de la importancia de que el director demuestre interés al contenido curricular (7), que es parte de su liderazgo dentro de la facultad; y la exigencia académica (12), la cual ubica al director como guardián de la exigencia académica y, por lo tanto, como defensor de la calidad institucional.

\section{Curriculum para la Orientación a los Alumnos}

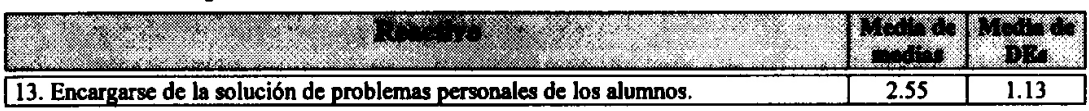

En esta categoría contamos con un solo reactivo, donde la media de las respuestas se ubica en el grado de la falta de importancia de la actividad, en gran parte por la también falta de consenso. Sin embargo, esta media por muy poco se ubica como actividad que el director no debería emprender. Nuestra inferencia de esto es que, si la facultad como institución se preocupara por los problemas personales de los alumnos (lo cual en todo caso no está establecido), esta actividad correspondería a alguien que no fuera el director.

\section{Ethos}

\begin{tabular}{|l|c|c|}
\hline & & \\
\hline $\begin{array}{l}\text { 14. Convivir con los alumnos, aun cuando conduzca al consumo de bebidas } \\
\text { alcohólicas. }\end{array}$ & 1.41 & 0.79 \\
\hline $\begin{array}{l}\text { 15. Propiciar un trabajo colectivo por parte de los docentes. } \\
\text { 16. Despedir a personal que carezca de ética profesional, aun cuando sean sus } \\
\text { amigos. }\end{array}$ & 4.61 & 0.70 \\
\hline 17. Respetar posturas de profesores antagónicos a la suya, sin presionarles a cambiar. & 4.62 & 0.96 \\
\hline 18. Tener una perspectiva de lo mexiquense. & 3.90 & 1.18 \\
\hline 19. Tener capacidad demagógica para poder convencer a los inconformes. & 3.00 & 1.00 \\
\hline 52. Permitir la negociación de sus calificaciones por parte de alumnos especiales. & 1.45 & 0.87 \\
\hline
\end{tabular}

Dentro de esta categoría de respuestas, encontramos que más de la mitad de los reactivos tienen carácter obligatorio o prohibitivo y que, además, éstas cuentan con un elevado grado de consenso. Uno de estos reactivos habla claramente acerca del control de la ética de los profesores en general (16). El otro habla acerca de la promoción de un espíritu participativo y colaborativo entre los profesores (15), lo cual implica la participación y liderazgo del director en el mismo sentido. 
De los reactivos que encuentran un rechazo muy fuerte, uno tiene que ver con la convivencia entre alumnos y el consumo de bebidas alcohólicas (14). Como éste es un reactivo híbrido polarizado, nuestro supuesto es que el rechazo se dirige hacia el consumo de alcohol, más que la convivencia en sí. Podemos especular acerca de las razones que explicaran este resultado; tal vez tengan que ver con la conveniencia de que el director mantenga su imagen como figura remota, la cual se pudiera perder si una convivencia llegara a ser demasiado desinhibida.

El otro que encuentra un rechazo fuerte habla acerca de la negociación de calificaciones y un favoritismo y discriminación entre los alumnos (52). Este tipo de acciones y actitudes por parte del director es altamente reprobado, lo cual significa para nosotros una clara preferencia por un trato equitativo y una honradez en la toma de decisiones.

De los dos reactivos que encuentran apoyo, pero sólo en el grado de deseabilidad y no obligatoriedad, el primero tiene que ver con la actitud respetuosa del director frente a posturas antagónicas a la suya (17). En este caso encontramos también la falta de un consenso entre los encuestados. Tal vez la explicación pudiera caer dentro de la naturaleza de las cuestiones en discrepancia; por un lado, cuestiones puramente académicas deberían ser respetadas, mientras otras cuestiones de tipo político interno o de relaciones humanas tal vez deberían sujetarse a presiones para transformarse.

El otro reactivo deseable sin ser obligatorio tiene que ver con la dimensión mexiquense (18). La cuestión aquí es cómo sería la contraparte de esta proposición. El no tener una perspectiva de lo mexiquense implicaría una desvinculación con el contexto inmediato y los problemas que enfrenta la sociedad local; al mismo tiempo, el tener esta visión local no implica necesariamente el excluir una perspectiva más amplia complementaria.

El reactivo que permaneció abierto habla acerca de la capacidad demagógica del director (19); otra vez ésta es una pregunta híbrida. Por una parte, es conveniente que el director convenza a sus compañeros de la facultad para que trabajen conjuntamente para alcanzar los objetivos institucionales y esto pudiera provocar una aceptación por parte de los encuestados. Por otra parte, la demagogia no es necesariamente el mecanismo más indicado dentro de un ámbito académico para conducir a otros. Por lo tanto, no es de sorprender que 
la media se ubicara completamente en el punto medio y que hubiera una amplia diversidad en las respuestas. Es decir, no existe ningún acuerdo sobre el mérito respectivo de esta actividad y esta característica.

\section{E. Recursos}

\begin{tabular}{|c|c|c|}
\hline (3) & Wompen & Worlon \\
\hline 21. Buscar financiamiento de la empresa privada. & 3.87 & 1.08 \\
\hline 22. Otorgarse la libertad de designar el uso de recursos. & 2.75 & 1.41 \\
\hline $\begin{array}{l}\text { 23. Informar de manera precisa al consejo de gobiemo acerca del manejo de los } \\
\text { recursos. }\end{array}$ & 4.76 & 0.58 \\
\hline $\begin{array}{l}\text { 24. Acudir solamente a criterios imparciales (como tasas de titulación y avances de } \\
\text { investigación) para justificar la búsqueda de más recursos de la Universidad. }\end{array}$ & 2.94 & 1.43 \\
\hline 25. Gastar dinero de la institución para eventos sociales. & 1.82 & 0.99 \\
\hline $\begin{array}{l}\text { 26. Gratificar a políticos importantes creando los espacios para su lucimiento con } \\
\text { fondos de la facultad. }\end{array}$ & 1.27 & 0.70 \\
\hline 27. Encubrir desfalcos e irregularidades para proteger la buena fama de la facultad. & 1.25 & 0.79 \\
\hline 28. Cobrar siempre cualquier trámite burocrático. & 1.89 & 1.21 \\
\hline
\end{tabular}

De los ocho reactivos en esta categoría, cuatro propuestas fueron rechazadas, dos se ubicaron en un punto intermedio y dos encontraron aceptación.

De éstas, hubo un apoyo muy fuerte, por lo que proponía que el director debería informar siempre a los consejos acerca del uso de los recursos de la facultad (23), lo cual significa una exigencia para un control - o por lo menos la exigencia a cuentas - sobre el director en cuanto a los recursos institucionales. En el mismo sentido, se rechazan otras proposiciones acerca del uso de dinero para eventos sociales (25), aunque éstos pudieran fomentar buenas relaciones humanas; y para gratificar a políticos importantes (26), aunque éstos pudieran contribuir más recursos para la facultad. Al mismo tiempo, se rechaza de manera muy determinante el encubrimiento de cualquier desfalco (27), lo cual sería de esperar, a pesar de que el instrumento tratara de matizar la respuesta, hablando acerca de la buena fama de la institución. Estos cuatro reactivos, considerados conjuntamente, refuerzan la idea de la universidad como un espacio ético sujeto a su normatividad, con lo cual necesariamente se identifica la figura del director de facultad.

Curiosamente, hay otro reactivo que propone la libertad o discrecionalidad de director para gastar dinero (22). Para que la respuesta ante esta pregunta fuera consistente con lo anterior, uno esperaría un rechazo por parte de los encuestados. Aunque se puede 
notar una tendencia en este sentido, finalmente resulta que el juicio colectivo se ubica en la falta de importancia, aunque la alta desviación estándar significa que este reactivo carece de consenso.

Otro reactivo interesante es el que habla acerca del cobro por trámites (28), porque en este caso se nota un rechazo ante la figura de un director quien solamente piense en los recursos como una finalidad en sí y quien no contemplara las necesidades de otros y la dimensión humana de su trabajo.

Aunque el juicio colectivo también determina que es deseable que el director busque recursos de la empresa privada (21), se demuestra cierta debilidad en el apoyo que se le da, tal vez por la suspicacia tradicional del académico hacia una vinculación demasiado estrecha con el sector productivo. Asimismo, se encuentra en el grado de imparcialidad (aunque con un elevado grado de desacuerdo) la proposición que el director se apegara solamente a criterios imparciales para conseguir más apoyo (24). Combinando estos dos reactivos, encontramos que muchos encuestados consideran que el director debe allegarse fondos para su proyecto académico, sin que les importe mucho cómo ni de dónde; mientras que otros temen a los compromisos implícitos en la aceptación de dinero de fuentes interesadas o por mecanismos no totalmente transparentes. A final de cuentas, no hay consenso sobre esto, lo cual permite cierta libertad de acción al director.

\section{F. Planeación y Organización}

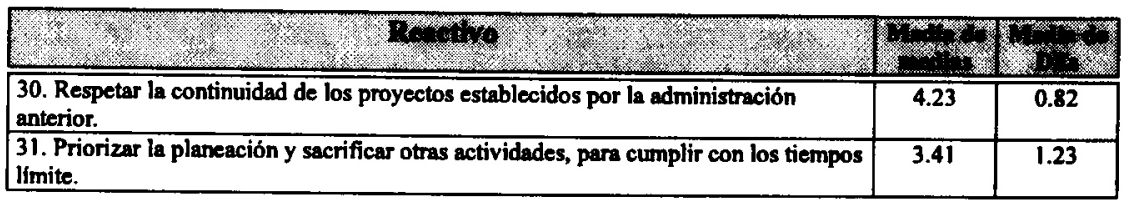

Tradicionalmente, la planeación y organización de cualquier institución son actividades medulares para su director. Sin embargo, sentimos de manera personal que estas actividades tienen menos importancia de lo que sugieren algunos autores. En este caso, tenemos solamente dos reactivos, de los cuales uno cuenta con cierto grado de apoyo, mientras el otro permanece sin decisión.

En el primer caso, la continuidad del proyecto institucional ya establecido (30) va en contra de la tradición mexicana de constantes 
cambios sexenales, frecuentemente por el capricho del político en turno; y por supuesto esta postura es mucho más sana. Esto implica el otorgamiento a los consejos de la facultad su debido reconocimiento como los órganos permanentes, otorgadores de continuidad, frente al director quien tiene cierta transitoriedad. Viendo esto desde la perspectiva del rol del director, se debe entender como una limitante a las decisiones unipersonales; se considera que hay otras instancias más importantes que el director, para el buen funcionamiento institucional.

En el otro caso, de nuevo se cuenta con un reactivo híbrido, donde cada uno de los componentes probablemente contaría con mucho respaldo. Sin embargo, en este caso encontramos que la cuestión de la importancia respectiva de la planeación por una parte y "otras actividades" y el respeto por los tiempos por otra (31) es una que permanece indeterminada. Cada punto de vista parece contar con sus defensores.

\begin{tabular}{|c|c|c|}
\hline 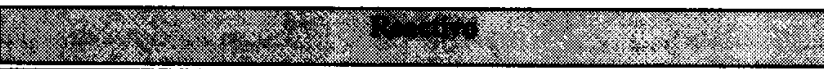 & (x) & $3+3 x^{3}$ \\
\hline 33. Delegar la atención a personal docente en el secretario académico. & 3.21 & 1.38 \\
\hline 34. Supervisar personalmente la actividad docente, observando clases. & 3.62 & 1.22 \\
\hline 35. Fomentar la libertad de pensamiento y de acción de profesores e investigadores. & 4.65 & 0.77 \\
\hline $\begin{array}{l}\text { 36. Mantener un equilibrio en la planta docente, invitando a trabajar a maestros de } \\
\text { afuera. }\end{array}$ & 4.21 & 0.91 \\
\hline 37. Contratar a los docentes por sus relaciones políticas. & 1.46 & 0.73 \\
\hline $\begin{array}{l}\text { 38. Acudir solamente a los docentes que le apoyaron en campania para formar su } \\
\text { equipo. }\end{array}$ & 1.34 & 0.78 \\
\hline $\begin{array}{l}\text { 39. Mantener a doctores y maestros en la institución, aun cuando no sean de su } \\
\text { equipo. }\end{array}$ & 4.29 & 1.16 \\
\hline 57. Promover la contratación preferencial de egresados de la facultad. & 3.66 & 1.14 \\
\hline
\end{tabular}

De los ocho reactivos en esta categoría, solamente uno se ubica cerca del punto medio (el que habla acerca de la delegación de cierta responsabilidad al secretario académico: 33), indicando que no es una cuestión sin importancia para el desempeño del rol del director, sino que no existe ningún consenso y las respuestas son muy dispersas.

En contraste, el fomento de libertad de acción entre los profesores (35) se define como obligatorio para el director, esto se asocia con la idea de la universidad como un espacio que promueve la libertad de pensamiento y trabajo entre los académicos.

En el mismo espíritu, dos proposiciones se encuentran totalmente prohibidas: la contratación de profesores por sus relaciones políticas 
(37) y el involucramiento en su equipo de trabajo solamente a esos profesores que apoyaran su campaña (38), lo cual evidentemente implica un respeto hacia otras posturas. Este es un tema que se repite con bastante frecuencia en este estudio.

Los otros cuatro reactivos son más bien de carácter deseable: que el director observe clases (34); que mantenga un equilibrio en su planta docente (36); que contrate a egresados de manera preferencial (57) y que mantenga dentro del plantel a profesores de alto nivel académico (39), aun cuando no sean de su equipo original.

Muchos de este grupo de reactivos se relacionan con la priorización de cualidades académicas y el proyecto institucional, la subordinación de otros intereses (como los políticos) y el mantenimiento de la facultad como un espacio abierto, plural y equilibrado.

\section{H. Evaluación}

\begin{tabular}{|l|c|c|}
\hline & & \\
\hline
\end{tabular}

En esta categoría encontramos dos reactivos, donde la muestra de encuestados expresa su juicio colectivo en el sentido de que estas actividades no tienen importancia para el rol del director; y otra vez este juicio se asocia con una gama amplia de respuestas demostradas por las elevadas desviaciones. Otra vez, esta situación se asocia con reactivos híbridos, donde cada parte se puede justificar fácilmente, pero donde los encuestados tienen dificultad colectivamente por decidir entre ellos.

Los dos otros reactivos encuentran apoyo en el grado de deseabilidad. Uno de estos dos también es híbrido (el que contrapone la evaluación permanente y cambios constantes: 41), pero en este caso los encuestados encontraron la posibilidad de optar por el lado de la evaluación. El cuarto, que propone la participación de todos en la evaluación institucional (43), otra vez enfatiza el carácter democrático y participativo de una dirección de facultad. 


\section{Archivos}

\begin{tabular}{|c|c|c|}
\hline (1) & 10 & Wringen \\
\hline 44. Vigilar estrictamente el manejo eficiente de los archivos. & 4.11 & 1.14 \\
\hline 45. Revisar detalladamente todos los documentos que tiene que firmar. & 4.68 & 0.76 \\
\hline 46. Informarse de las estadisticas a través del departamento de control escolar. & 4.55 & 0.80 \\
\hline
\end{tabular}

Un apoyo decidido por estos tres reactivos asocia al director plenamente con cuestiones administrativas de la facultad. Y de hecho, éste es el caso. Dos de ellos tienen carácter de obligatoriedad: la revisión detallada de todos los documentos (45) y el monitoreo de las estadísticas de control escolar (46). El otro, que tiene que ver con la supervisión de los archivos (44), resulta ser menos fuerte, pero no obstante sigue como algo que es deseable que haga el director.

Efectivamente, estas respuestas nos dicen claramente que el rol del director tiene una dimensión como jefe de la administración, que es muy importante.

\section{J. Motivación}

\begin{tabular}{|c|c|c|}
\hline S. & (28, & (3) \\
\hline $\begin{array}{l}\text { 47. Perdonar a los alumnos que incurren en violaciones menores de las leyes y } \\
\text { reglamentos. }\end{array}$ & 2.54 & 1.34 \\
\hline 48. Salvaguardar los derechos individuales de los estudiantes frente a los profesores. & 4.23 & 1.17 \\
\hline $\begin{array}{l}\text { 49. Respetar los criterios institucionales, aun cuando impidan el alcance de metas de } \\
\text { superación. }\end{array}$ & 2.94 & 1.36 \\
\hline $\begin{array}{l}\text { 50. Trabajar más allá de su horario normal para motivar a la comunidad con su } \\
\text { ejemplo. }\end{array}$ & 3.79 & 1.18 \\
\hline 51. Dar importancia al trabajo de todos los integrantes de la organización. & 4.75 & 0.59 \\
\hline 53. Cambiar a los profesores cuando asi lo demanden los alumnos. & 2.97 & 1.25 \\
\hline 54. Emplear su criterio personal para otorgar reconocimiento a algumos profesores. & 2.16 & 1.24 \\
\hline
\end{tabular}

Los reactivos agrupados dentro de esta categoría, hablan acerca del personal en todos los niveles. El que encuentra mayor respaldo (a grado de obligatoriedad) es el que quiere reconocer la importancia de todos (51) para avanzar el proyecto institucional. Este reactivo además cuenta con un elevado grado de consenso. Otra vez tenemos un reactivo que implica un estilo inclusivo y colaborativo de liderazgo.

Los tres reactivos restantes se ubican en el grado de lo deseable, más que lo esencial. La defensa de los estudiantes frente a posibles imposiciones por parte de los profesores (48) se relaciona con 
cuestiones de justicia, definiendo así al director como un tipo de procurador. El acudir al ejemplo personal trabajando más horas de las contractuales para motivar a la gente (50) sugiere un deseo para identificar al director como parte de un equipo. Y el rechazo del uso de criterios personales para motivar a la gente (54) ubica al rol del director dentro de la normatividad institucional.

A los tres reactivos restantes no se les otorga importancia en la definición de rol. Otra vez esto se explica con base en que todos son híbridos, y los encuestados demuestran su falta de consenso sobre la relativa importancia de cada parte.

\section{K. Capacitación de Personal}

\begin{tabular}{|c|c|c|}
\hline $\begin{array}{l}\text { 55. Implantar cursos de actualización obligatorios para profesionalizar a sus cuadros } \\
\text { docente y de investigación. }\end{array}$ & 4.43 & 0.94 \\
\hline 56. Dar orientación personalmente a los profesores de nuevo ingreso. & 4.19 & 0.90 \\
\hline
\end{tabular}

Esta categoría se encuentra con pocos reactivos, sugiriendo desde un inicio que muy probablemente este grupo no sería medular dentro del rol de director de facultad. Sin embargo, resulta que los dos encuentran un nivel de apoyo que los ubica en el grado de lo deseable.

Esto puede ser porque el primero, que busca la promoción de cursos de capacitación (55), no implica que el propio director imparta el curso; mientras el segundo, acerca de la orientación de profesores de nuevo ingreso (56) es de corta duración y a lo mejor tiene que ver con una orientación acerca de los espacios físicos o de las normas administrativas, más que una orientación dirigida hacia la didáctica.

\section{Resolución de Conflictos}

\begin{tabular}{|l|c|c|}
\hline \hline & & \\
\hline
\end{tabular}


En esta categoría encontramos siete reactivos, de los cuales dos encuentran fuerte apoyo, mientras tres tienen el grado de deseabilidad; dos más se ubican en el grado donde no importa si el director lo haga o no; y otra vez más encontramos que esto se debe más que nada a la dispersión de las respuestas.

El reactivo que propone que el director debe tomar en cuenta el peso político de ciertos grupos (62) es interesante porque, por una parte, se puede ver la polarización de opiniones entre los que reconocen la realidad, donde es conveniente tomar en cuenta a ciertas personas para mantenerles "a bordo"; y por otra parte, la opinión contraria que, suponemos, prefiere que se apegue solamente a criterios académicos.

El otro reactivo en el mismo grupo habla acerca del manejo discreto de los puntos conflictivos (63), bajo el supuesto de que el director pueda avanzar el proyecto institucional, lo cual nos parecía personalmente, una propuesta que hubiera encontrado respaldo. Sin embargo, en general no fue así. Debemos suponer que la minoría que estuviera en contra está indicando su preferencia por una solución negociada abiertamente dentro de los órganos colegiados de la facultad.

Tal vez esta interpretación encuentre respaldo en las respuestas a los otros reactivos de esta categoría. La creación de canales para la expresión de inconformidades (60) y el análisis profundo de los problemas (59), los cuales tienen carácter de obligatoriedad, ambos sugieren una búsqueda de apertura dentro de la institución, para que se tomen en cuenta los puntos de vista de todos los involucrados.

De manera similar, el apoyo para la proposición de que el director debe negociar su propia postura (61) y que acuda siempre al reglamento (64) indica la conveniencia de encontrar soluciones a conflictos a través de mecanismos institucionales que vayan más allá de lo personal. El rechazo a la proposición de que el director debe acudir a otros mecanismos para conseguir la conformidad de los profesores (58) refuerza esta interpretación.

Como se observa abajo, esta categoría es una de las que más reactivos tiene. De éstos, dos son considerados como obligatorios, mientras seis de los otros son deseables. 
M. Comunicación Interna

\begin{tabular}{|c|c|c|}
\hline (3) & Wention & Ting, \\
\hline $\begin{array}{l}\text { 29. Estar enterado, de manera directa, de la problemática de la facultad, a través de } \\
\text { visitas constantes a todas las instancias internas. }\end{array}$ & 4.80 & 0.61 \\
\hline 65. Sondear formalmente a la comunidad sobre toda cuestión importante. & 4.48 & 0.77 \\
\hline 66. Permitir el acceso abierto a información confiable. & 3.66 & 1.44 \\
\hline 67. Visitar a los alumnos en sus salones para intercambiar información directa. & 4.56 & 0.67 \\
\hline 68. Compartir toda su información con los consejos. & 4.25 & 0.98 \\
\hline 69. Enviar su información y publicaciones a las oficinas de Gobiemo. & 3.14 & 1.35 \\
\hline 70. Crear redes de informantes para conocer lo que la gente realmente piensa. & 3.56 & 1.29 \\
\hline $\begin{array}{l}\text { 71. Permitir la existencia de medios de comunicación independientes y críticos, aun } \\
\text { cuando hablen mal de su administración. }\end{array}$ & 4.14 & 1.04 \\
\hline $\begin{array}{l}\text { 72. Asistir a casi todos los informes de otras facultades, para mantener su presencia } \\
\text { dentro de la universidad. }\end{array}$ & 3.66 & 1.15 \\
\hline
\end{tabular}

Los dos obligatorios son muy similares, en el sentido de que uno propone que el director debe visitar todas las instancias de la facultad (29), mientras el otro es más específico, hablando acerca de visitas a los salones para comunicarse con los alumnos (67), en ambos casos con la finalidad de acceder directamente a información. Esos mecanismos deberían ser vistos como directos pero informales.

Otro reactivo piensa en mecanismos más formales, hablando acerca del sondeo de la comunidad sobre cuestiones importantes (65). También apoyado por los encuestados es el reactivo que cuestiona la creación de redes de informantes dentro de la institución (70). Aunque este reactivo es considerado como deseable, lo es solamente de manera muy marginal. Una aclaración al respecto es que aquí hemos sustituido un lenguaje más moderado por las palabras de un entrevistado, quien hablaba acerca de redes de espionaje en una administración que conocía. La idea evidentemente es la misma, pero la expresión es positiva. En este caso vemos que la idea cuenta con apoyo, aunque hay que reconocer que aun con la redacción moderada, muchos encuestados se sienten suspicaces ante esta proposición. A lo mejor prefieren canales de comunicación más abiertos y transparentes.

Todavía sobre la comunicación, pero ahora en dirección contraria de su transmisión, un reactivo también considerado como deseable busca el acceso a información confiable (66), mientras otro enfoca la transmisión de información a los consejos (68). En estos casos, estamos viendo al director en su otra función, como transmisor de información dentro de la institución. 
En un sentido un poco diferente, se encuentra apoyo para la proposición acerca de la existencia de medios de comunicación críticos e independientes (71). Este juicio se puede relacionar con los otros que hablan (arriba) acerca de la conveniencia de posturas diferentes dentro de la facultad.

Los dos reactivos restantes pueden ser considerados como "comunicación interna" solamente si se le otorga una definición más amplia a la institución. Así es que la presencia del director en eventos de otras facultades (72) es considerada deseable, pero sólo marginalmente así; y tal vez la segunda parte del reactivo pone una glosa en esa asistencia diferente a la de comunicación.

En contraste, la comunicación con oficinas de gobierno (69), entendiendo en este caso a la universidad como un organismo descentralizado del gobierno, encuentra una respuesta mixta que da como resultado un juicio indeterminado para el rol del director.

\section{N. Edificios}

\begin{tabular}{|l|c|c|}
\hline & & \\
\hline $\begin{array}{l}\text { 73. Supervisar personalmente la condición de los salones, bafos, biblioteca y } \\
\text { edificios. }\end{array}$ & 4.23 & 0.98 \\
\hline $\begin{array}{l}\text { 74. Dar prioridad al buen estado de todos los edificios e instalaciones, más que a } \\
\text { becas y libros. }\end{array}$ & 2.35 & 1.19 \\
\hline $\begin{array}{l}\text { 75. Procurar la utilización al cien por ciento de las instalaciones, aun cuando esto } \\
\text { signifique grupos numerosos en los primeros años. }\end{array}$ & 2.54 & 1.32 \\
\hline
\end{tabular}

En esta categoría de reactivos no hay ninguno que se ubique en los extremos. Es deseable, pero nada más, que el director supervise personalmente las instalaciones (73), pero las condiciones físicas de éstas no deberían recibir mayor prioridad de su parte que aspectos de apoyo para el trabajo académico como libros y becas (74).

Resulta como neutral el reactivo que contrasta el uso de las instalaciones y la presencia de grupos numerosos (75), porque estas dos opciones cuentan cada una con apoyo entre algunos encuestados. Para el rol del director, la polarización está entre la eficiencia administrativa y la eficiencia académica, algo que los encuestados no pueden decidir. 


\section{O. Docencia e Investigación}

\begin{tabular}{|l|c|c|c|}
\hline 76. Definir líneas de investigación y dificultar la libre selección de otras. & . & . \\
\hline 77. Preocuparse por la calidad de las tesis, aun a costo de reprobar a algunas. & 2.29 & 1.35 \\
\hline 78. Seguir ejerciendo personalmente la docencia y la investigación. & 4.03 & 1.18 \\
\hline
\end{tabular}

Esta categoría de respuestas es interesante, porque pretende identificar qué tanto es importante que el director dé un liderazgo a través de su ejemplo, identificándose todavía con las actividades esenciales de la facultad. Encontramos que esto no se define como obligatorio, aunque sí es deseable.

Medular en la exploración de este conjunto de reactivos es el que cuestiona el ejercicio del director como docente e investigador (78), el cual encuentra apoyo, aunque también se debe notar que este juicio no cuenta con un consenso, ubicándose su desviación estándar arriba del 1.0 .

Tal vez el reactivo que habla acerca de una exigencia frente a la calidad de las tesis debería ubicarse en la categoría de lo académico, pero lo hemos incluido aquí porque suponemos que el director se involucra activamente en el proceso de titulación (o por lo menos que se interesa mucho). Por lo tanto, es otra actividad donde el director puede dar un liderazgo activo en el quehacer académico de la facultad.

El tercer reactivo donde el director pudiera desempeñarse activamente es en la definición y prohibición de líneas de investigación (76), el cual se encuentra rechazado, muy proba-blemente por la inclusión de la idea de prohibición de ciertas líneas. Esto habla acerca de la libertad de academia de los profesores y una resistencia ante la idea de una imposición en su actividad. A lo mejor, un acuerdo consensual para precisar líneas sería aceptable.

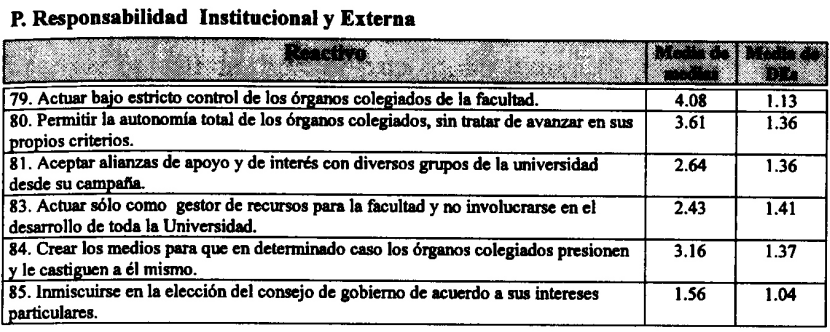


De los seis reactivos en esta categoría, encontramos que dos tienen apoyo y dos rechazo, todos en el grado de deseabilidad; mientras que hay dos más que terminan en la imprecisión.

Los dos que encuentran respaldo hablan acerca de los consejos de la facultad, tanto como organismos que controlan las acciones del director (79) como los que tienen una autonomía (80). Con estas repuestas vemos el deseo para contar con decisiones colegiadas, el cual va en contra de la visión del director como el tomador de decisiones de manera unipersonal.

De los dos reactivos rechazados, uno defiende el proceso democrático de la elección del consejo sin presiones sobre el director (85), lo cual refuerza la idea de la autonomía de los consejos y la definición del rol del director como el ejecutivo de sus decisiones, no como manipulador o controlador de ellas.

El otro, que cuestiona el involucramiento del director en la toma de decisiones a nivel de universidad (83), contrasta esto con una actividad más limitada como gestor de recursos. En este caso, el rechazo significa que el director debe participar en el proceso de desarrollo de toda la universidad; o sea, que tiene una responsabilidad en este rumbo. Sin embargo, se debe notar la falta de consenso sobre este punto expresada a través de una desviación estándar elevada, lo cual significa que algunos de los encuestados consideran que la prioridad del director es la de desempeñarse en un espacio más reducido, como es el de la facultad.

Los dos reactivos que no encuentran ningún consenso (y por lo tanto se ubican en el grupo de reactivos abiertos para el albedrío del director sobre si los emprende o no) tienen que ver con la formación de alianzas con grupos dentro de la universidad (81); lo cual pudiera ser interpretado como la creación de obligaciones y de responsabilidades hacia personas ajenas a la facultad; y con la creación de mecanismos para dar más poder a los colegios de la facultad, para que puedan sancionar (y por lo tanto controlar más) al propio director (84). La falta de consenso sobre este segundo reactivo es interesante, porque hemos visto en varios casos la expresión de un deseo de que los colegios sean independientes del director; en cambio, cuando se presenta un reactivo que pretenda el establecimiento de mecanismos para reforzar esta autonomía, el consenso no se nota. A lo mejor hay que explorar más los límites dentro de este equilibrio de fuerzas y lo que significan para la 
práctica. Hasta ahora, parece que tenemos una situación donde el director respeta la autonomía de los consejos; pero que éstos no tienen ninguna fuerza para obligar al director a que lo haga.

\section{Q. Comunidad y Sociedad}

\begin{tabular}{|c|c|c|}
\hline (1) & (I) & (19: \\
\hline $\begin{array}{l}\text { 86. Ampliar el contacto con grupos populares para que la facultad resuelva sus } \\
\text { problemas. }\end{array}$ & 2.76 & $\overline{1.36}$ \\
\hline 87. Vincularse sólo con aquellos sectores que le puedan dar prestigio a la facultad. & 2.65 & 1.36 \\
\hline 88. Relacionarse con el gobierno sólo para proyectos rentables & 2.63 & 1.28 \\
\hline nte prácticas profesionales en el sector p & 3.60 & 1.41 \\
\hline 90. Ofrecer servicios de consultorias al sector productivo. & 4.31 & 1.01 \\
\hline
\end{tabular}

En esta categoría de reactivos encontramos tres donde el juicio colectivo es que la actividad no es de importancia para la precisión del rol del director; mientras que dos más son considerados como deseables, pero no como esenciales. Los cinco reactivos cuentan con una desviación estándar por arriba del 1.0.

El reactivo que cuenta con mayor respaldo tiene que ver con el ofrecimiento de consultorías al sector productivo (90). Esto se relaciona con la captura de ingresos para la facultad, con un apoyo para los estudiantes a través de una vinculación con este sector y tal vez también representa una parte de la dimensión profesional del rol.

También con apoyo, pero casi cayendo en la ambigüedad, es el reactivo que habla acerca de la negociación para las prácticas profesionales (89) por parte del director. En general, podemos entender esto como el aspecto de su rol relacionado con la vinculación externa; pero el grupo que le niega esta función, tal vez considere que corresponde a otra persona de su equipo.

Los otros tres reactivos hablan acerca de la relación de la facultad con grupos populares (86), con sectores de prestigio (87) y con el gobierno (88) a través del director y todos caen en la indefinición. Por una parte, según la literatura, como cabeza visible de la institución, un director debe funcionar como el conducto con organismos externos. Sin embargo, resulta que aproximadamente la mitad de los encuestados rechaza esta función, lo cual implica que para ellos su función real se ubica dentro de la facultad y de la universidad. 


\section{R. Empleadores y Profesionalismo}

\begin{tabular}{|c|c|c|}
\hline 91. Poner en contacto a los egresados con sus posibles emplendores. & 4.42 & 0.80 \\
\hline 92. Mantener una organización con los egresados y usarla como una bolsa de trabajo. & 4.35 & 0.89 \\
\hline 93. Haber ejercido anteriormente la profesión y no haber sido un puro académico. & 4.23 & 1.01 \\
\hline
\end{tabular}

Estos tres reactivos tienen que ver con la dimensión profesional del director, definiéndose los tres como actividades deseables. Esto se expresa claramente en el que precisa el ejercicio profesional (93) como antecedente complementario al académico. Los otros dos tienen que ver con la ubicación laboral de los egresados, directamente con los empleadores (91) o a través de otros egresados (92). En el caso del primero de este grupo de reactivos, vemos una relación con uno de los anteriormente comentados (el 89), lo cual da fuerza a nuestra interpretación.

\section{S. Trabajo fuera de la Facultad}

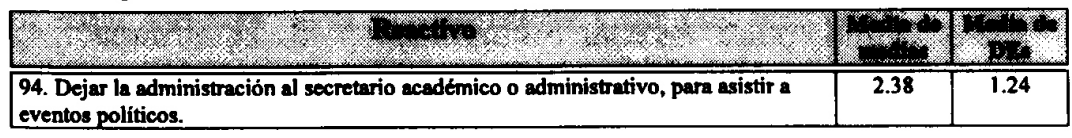

En esta categoría contamos solamente con un reactivo, lo cual sugiere desde un inicio que no se le otorga mucha importancia. En este caso, se ve una contrastación entre una delegación de actividades a los secretarios de la facultad y la propia asistencia del director en eventos políticos (94). En este caso se rechaza esta delegación de actividades, pero tal vez esto se deba a que la alternativa no es considerada como legítima tampoco (y en mayor grado).

\begin{tabular}{|c|c|c|}
\hline W & Torm 0 & $P^{2}$ \\
\hline $\begin{array}{l}\text { 32. Visitar algunas escuelas preparatorias para que se den cuenta de lo que hace la } \\
\text { Facultad. }\end{array}$ & 3.86 & 1.19 \\
\hline 69. Enviar su información y publicaciones a las oficinas de Gobierno. & 3.14 & 1.35 \\
\hline 82. Mantener un contacto personal con el Rector. & 4.45 & 0.83 \\
\hline 95. Ser militante de un partido politico. & 2.12 & 1.15 \\
\hline 96. Participar activamente en el colegio de su ramo. & 4.23 & 0.88 \\
\hline 97. Vincularse a otras instituciones académicas que tengan prestigio académico. & 4.50 & 0.77 \\
\hline $\begin{array}{l}\text { 98. Emplear recursos financieros de la facultad para cuidar su relación con los medios } \\
\text { de comunicación. }\end{array}$ & 1.81 & 1.15 \\
\hline $\begin{array}{l}\text { 99. Pertenecer a organizaciones internacionales prestigiadas para vincular a su } \\
\text { facultad. }\end{array}$ & 4.47 & 0.77 \\
\hline $\begin{array}{l}\text { 100. Tener libertad de comunicación hacia el exterior, sin tener que pasar por } \\
\text { instancias de Rectoria. }\end{array}$ & 3.60 & 1.43 \\
\hline
\end{tabular}


En esta categoría tenemos varios reactivos, lo cual indica potencialmente su importancia para el rol. Sin embargo, a final de cuentas resulta que solamente uno fue considerado como esencial, el cual habla acerca de la vinculación con otras instituciones de prestigio académico (97). En cambio, cinco son considerados como deseables y dos más lo son en sentido negativo, dejando indefinido solamente un reactivo.

Éste tiene que ver con las relaciones con el gobierno (69) y es un reactivo que comentamos anteriormente. En este momento nuestra interpretación es que algunas personas dan importancia a esta actividad, mientras otras prefieren guardar una clara separación entre la universidad y el gobierno.

Este segundo punto de vista encuentra respaldo en otro reactivo, donde el juicio colectivo rechaza la identificación del director con algún partido político (95). También se rechaza el uso de fondos de la facultad para influir en la publicación de noticias en los medios de comunicación (98), aunque otro reactivo (100) reconoce la importancia de mantener una relación directa con los medios. Evidentemente, lo que se rechaza es el uso de recursos de la facultad para hacerlo; no es legítimo.

Las otras actividades que se definen como deseables son el mantenimiento de una relación con otras instituciones: preparatorias (32), organizaciones internacionales (99), su colegio profesional (96) y el rector (82), entendiendo a este último como una "institución" ajena a la facultad. En todos estos casos, se ve al director como el representante de la institución frente a otras instancias. Se nota menos fuerza en el apoyo a nivel de la escuela preparatoria, tal vez porque ésta no cuenta con tanto prestigio, por lo cual la vinculación con ella pudiera ser una actividad emprendida por otra persona.

\section{Discusión y conclusiones}

Lo que emerge de este estudio es que la mayoría de los reactivos aportan elementos para precisar el rol del director. La mayoría habla acerca de actividades que un director debe hacer e incluso de las que tiene que hacer; y también en sentido negativo: actividades que debe evitar. Esto nos ayuda mucho para discernir los detalles del rol del director. 
De la presentación anterior, es posible ver claramente cuáles son las actividades que reciben mayor aceptación por parte de los grupos de encuestados (algunas veces en forma negativa, como un rechazo fuerte). Esto se ha comentado en el transcurso de este documento. Sin embargo, esta forma de ver los datos no nos permite detectar los patrones que pudieran emerger de ellos; los datos aislados no conducen a una comprensión teórica acerca del rol.

Al inicio planteamos que una forma de definir la importancia de las diferentes categorías de actividad sería a través del número de reactivos que tuvieran; y ya examinamos esto al inicio de este análisis. Según esta forma de abarcar la problemática, encontramos que cuatro de las categorías medulares tienen que ver con actividades internas de la facultad: las de comunicación interna, el currículum académico, los recursos y el manejo del personal docente. En cambio, hay dos categorías importantes (por su número de reactivos) que tienen que ver con la responsabilidad externa e institucional; y las relaciones con instituciones externas.

Por una parte, esto pudiera representar una manera de distinguir las actividades más importantes que integran el rol del director de facultad en la universidad mexicana. Por otra, pudiera indicar que son las actividades que resaltan más para nuestros informantes, tal vez porque su realización sea algunas veces inadecuada. Nos quedamos con esta duda.

Como alternativa, la detección de las categorías más importantes también pudiera hacerse a través del puntaje ponderado que se asigna a cada una (osea la media de los puntos para cada categoría); aunque en este caso debemos reconocer que pudiera existir un sesgo en la interpretación por la ubicación de ciertos reactivos en cierta categoría y no en otra. Este es un riesgo que debemos reconocer, aunque algunas veces en este texto hemos tratado de resaltar abiertamente la dificultad y resolviéndola a través de la contemplación de un reactivo en más de una categoría, cuando pareciera relevante hacerlo. Pero a final de cuentas, este acercamiento no parece muy satisfactorio, porque depende demasiado de un juicio subjetivo sobre la asignación de los reactivos a categorías.

Más convincente, quizás, es el examinar las categorías de actividad donde existe un reactivo o más que tengan un elevado puntaje de aceptación o de rechazo. A manera de ejemplo, la primera categoría 
(A: identificación de metas) cuenta con tres reactivos que se rechazan (aunque ligeramente) y uno que se define como indiferente; pero en cambio, cuenta también con el reactivo que cuenta con mayor apoyo en toda la batería. Con esta gama de respuestas ante los reactivos, no es posible ser categóricos sobre la importancia relativa de la identificación de metas por parte del director, según las opiniones de nuestros informantes.

Así es que este proceso tampoco nos ayuda mucho, porque casi todas las categorías contienen una gama de apoyo, rechazo e indiferencia ante los reactivos. Si dentro de cierta categoría un reactivo cuenta con apoyo, mientras todos los demás no, ¿qué podremos concluir realmente?

Lo que encontramos, entonces, en términos generales es que con excepción de la tercera categoría (que trata del currículum para la orientación de alumnos), no hay ninguna que no contenga mínimamente un elemento que aporte algo para definir el rol. Esto implica que todas las categorías tienen algo de importancia, pero que habrá que reflexionar más y seguir analizando de otras maneras para identificar los reactivos más importantes. La naturaleza de la actividad en sí parece insuficiente para precisar el rol.

\section{E-mail:jfarrand@coatepec.uaemex.mx}

\section{Bibliografía}

Banton, (1965), Roles, Londres.

Biddle. B,J y Thomas E., (1966), Role theory, Concepts and Research, Wile: Nueva York.

Farrand John, (1987), "An analysis of the tasks of headteachers and their deputies in twelve Mexican secondary schools and the implications for training", en Tesis doctoral, University of Bristol.

Farrand Rogers, John, (1993), La definición de roles dentro de algunas unidades universitarias desconcentradas, Documento interno, Toluca: Centro de Estudios Estratégicos, Universidad Autónoma del Estado de México.

Farrand Rogers, John, (1996), "El rol del director de facultad: cuestiones éticas no abiertas a discusión", en ponencia presentada en el Segundo Coloquio Regional de Investigación, Toluca: Universidad Autónoma del Estado de México.

García García, Gloria María Teresa y Mejía de Sánchez, María Guadalupe, (1990), Evaluación de la Práctica del Directivo Universitario de la UAEM y su Posible Perfil, Toluca: UAEM, Centro de Investigaciones en Ciencias Sociales y Humanidades.

Gross N, et al (1958), Explorations in role analysis: Studies of the school superintendancy role, Nueva York; Wiley. 
Hargreaves, DH (1972), Interpersonal relations and education. Londres; Routledge and Kegan Paul.

Ortiz Jiménez, Fernando y Farrand Rogers, John (1994), "La teoría de rol dentro de la sociología mexicana: un posible marco para interpretar el quehacer de un director de facultad", Ponencia presentada en el III Encuentro Estatal de Investigación Educativa. Querétaro.

Popitz H (1972), "The concept of social role as an element of sociological theory, en Jackson JA (1972)", en Role, Cambridge: Cambridge University Press.

Verona Casas, Gumaro (1996), "La orientación del Estatuto Universitario en la actividad del director de facultad", en ponencia presentada en el Segundo Coloquio Regional de Investigación, Toluca: Universidad Autónoma del Estado de México. 